\title{
MRI of the Psoas Major Muscle: Origin, Attachment, Anatomical Variants and Correlation with the Lumbar Disc Extrusion
}

\author{
Kakarala $\mathrm{A}^{1}$, Banitalebi $\mathrm{H}^{2}$, Borthne $\mathrm{AS}^{2}$ and Pierre-Jerome $\mathrm{C}^{2_{2}}$ \\ ${ }^{1}$ Department of Radiology, Emory University School of Medicine, Atlanta, GA, USA \\ ${ }^{2}$ Department of Radiology, Akershus University Hospital, Oslo, Norway
}

${ }^{*}$ Corresponding author: Pierre-Jerome C, M.D. PhD, Associate Professor of Radiology, Department of Radiology/ MSK Division, Akershus University Hospital, 75 Sykehusveien, 7500 Lorenskog, Norway, Tel: +47.679-69750, Cel: +47.47.641954, E-mail: clpi@ahus.no, claudepierrejerome7@gmail.com

Citation: Kakarala A, Banitalebi H, Borthne AS, Pierre-Jerome C (2016) MRI of the Psoas Major Muscle: Origin, Attachment, Anatomical Variants and Correlation with the Lumbar Disc Extrusion. J Adv Radiol Med Image 1(2): 202. doi: 10.15744/2456-5504.1.202

Received Date: June 30, 2016 Accepted Date: October 24, 2016 Published Date: October 25, 2016

\begin{abstract}
Purposes: 1) to verify the psoas major muscle (PMM) anatomical origin and variants, 2) to evaluate the PMM attachment to the lumbar disc and variants, and 3) to search for correlation between the anatomic variants of PMM attachment to the disc and disc extrusion.

Materials and Methods: Magnetic resonance examinations of the lumbar spine of 213 females and 170 males, mean age 60.3 years (range 15-85), were retrieved to study the PMM. In one-hundred and eighty-four subjects, the PMM attachment to the lumbar discs was reviewed, as we searched for disc extrusion. The prevalence of anatomical variants of PMM origin and the variants of PMM attachment to the disc was calculated. Spearman correlation coefficient was used to correlate the variants of the PMM attachment to the discs and disc extrusion.

Results: PMM originated mostly from the Th12-L1 disc, in 271 (70.8\%) subjects. Anatomical variants of PMM origin were found in onehundred-and-twelve (29.2\%) subjects. The PMM most caudal site of origin was at body L2, in 3 (0.7\%) subjects. Among the one-hundred and eighty-four subjects, the PMM did not attach to the L5-S1 disc in 173 (94\%) subjects. A higher prevalence of disc extrusion was detected at L5-S1 level, in 65 (35.3\%) subjects.

Conclusion: The PMM originates most frequently from the Th12-L1 disc. The absence of PMM attachment to the disc may contribute to disc extrusion. These findings deserve further investigations.
\end{abstract}

Keywords: Psoas Major Muscle; Anatomy; Anatomical Variants; MRI

\section{Introduction}

The Psoas major muscle (PMM) runs on both sides of the lumbar column originating from the thoracolumbar segment. Distally, the PMM joins the iliacus to form the iliopsoas muscle [1]. Viewed on the magnetic resonance (MR) coronal images of the lumbar region, the PMM major has a fusiform appearance with relative sharp borders and a symmetrical configuration, in most individuals (Figure 1). In less than fifty per cent of human subjects, the psoas major is accompanied by the psoas minor muscle $[1,2]$. This study focuses entirely on the anatomy and the anatomical variants of the PMM, amid the biomechanical role of the latter in the motion of the lumbar spine and the hip [3].

Anatomically, the PMM is made of two distinct layers: a superficial and a deep layer, which can be appreciated on MR axial images (Figure 2). The exact origin of the PMM varies. It may originate from Th11 or Th12 level, above or below [1-3,4]. Although the prevalence of these variants is literally unknown. Most of the reports [4-8] on the PMM focus on its biomechanical role in the stability and motility of the lumbar spine. There are no dedicated studies on the prevalence of the variants of the PMM origin. And the possible interference of these variants in the spine biomechanics has not been investigated.

From its origin to its junction with the iliacus muscle, the PMM attaches to the vertebral bodies, the transverse processes and the lumbar discs, on both sides [1-4,9]. Along with the back muscles, the PMM maintains the stability of the lumbar spine due to its bilateral attachments [10,11]. The infrastructure of the PMM is made of a series of overlapping segmental fascicles. At the disc level, these fascicles firmly attach bilaterally to the anterolateral surface of the disc's annulus fibrosus. They often extend posteriorly close to the intervertebral foramen [1-3,12]. Whether or not the PMM fibers provide support and protection to the lumbar discs has not yet been demonstrated. 


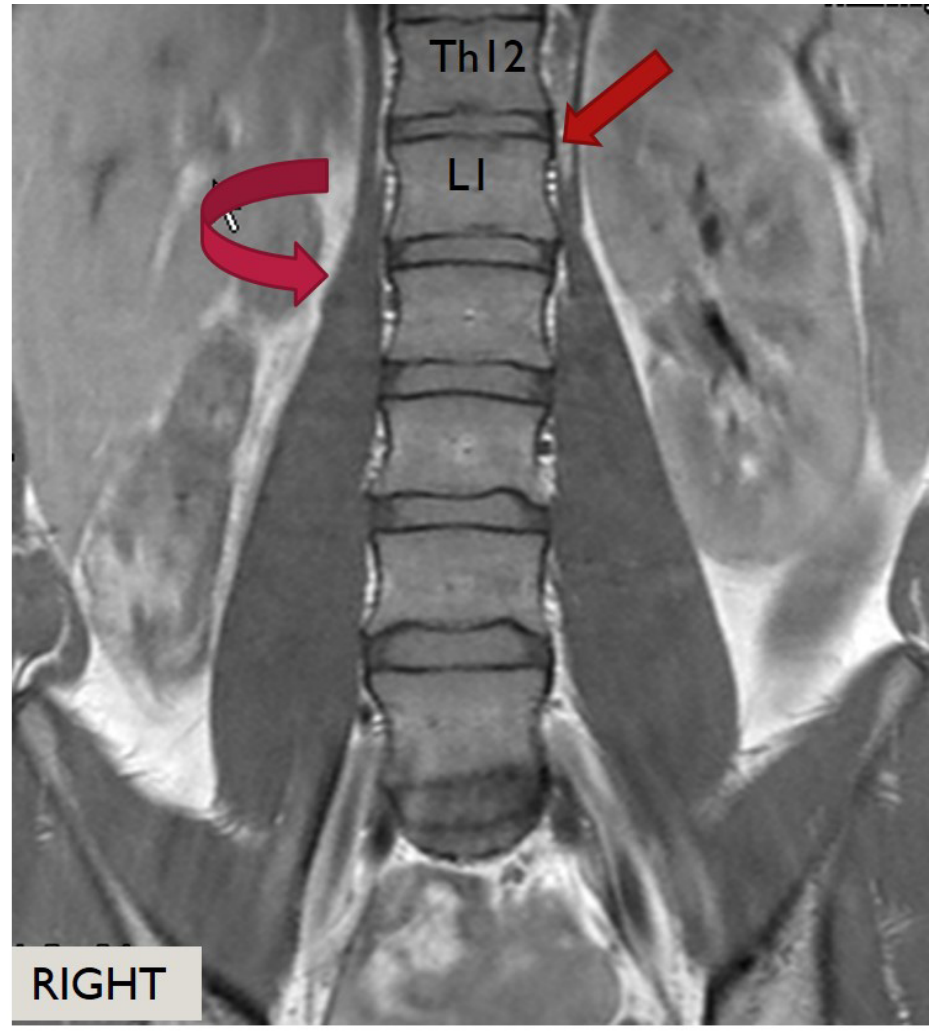

Figure 1A: MRI Turbo Spin Echo (TSE) proton density (PD) coronal image. Normal anatomical configuration of the PMM origin. The TSE PD coronal image displays the PMM (curved arrow), and the normal origin of the PMM from the Th12-L1 disc (arrow)

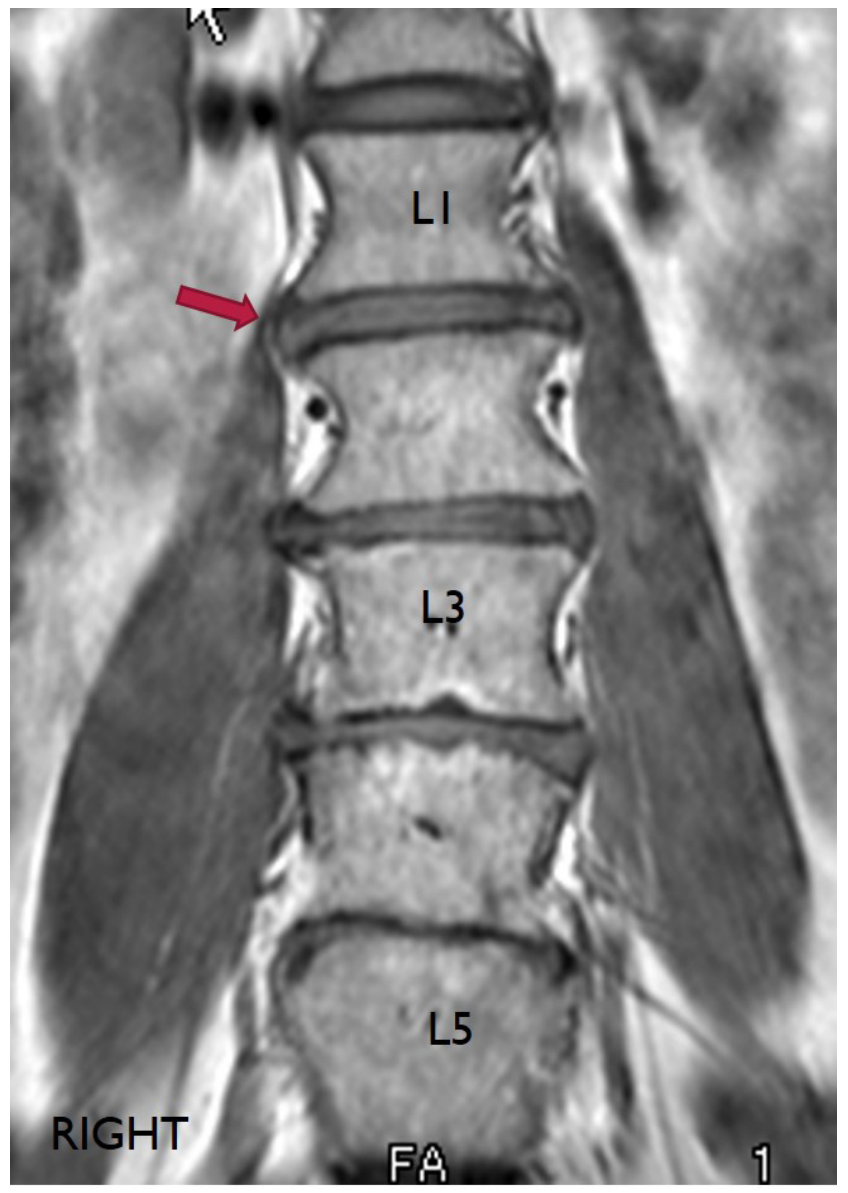

Figure 1B: MRI PD coronal image. Anatomical variant of the PMM origin. The PMM originates from the L1L2 disc on the right (arrow) 


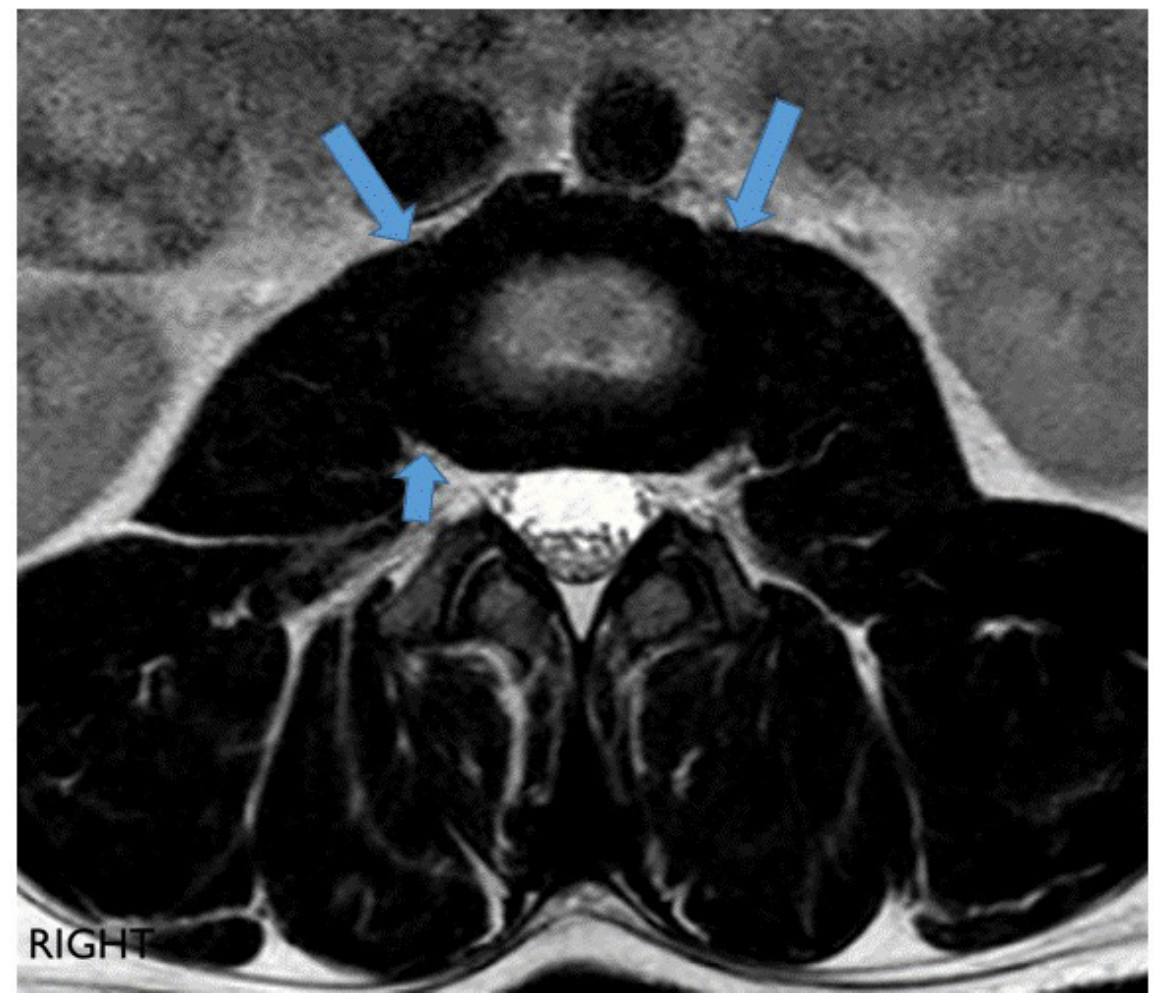

Figure 2A: MRI TSE T2 W axial image. Normal PMM attachment to the disc. The axial image of the L1-L2 disc shows the attachment of the PMM to the anterolateral border of the disc, bilaterally (arrows)

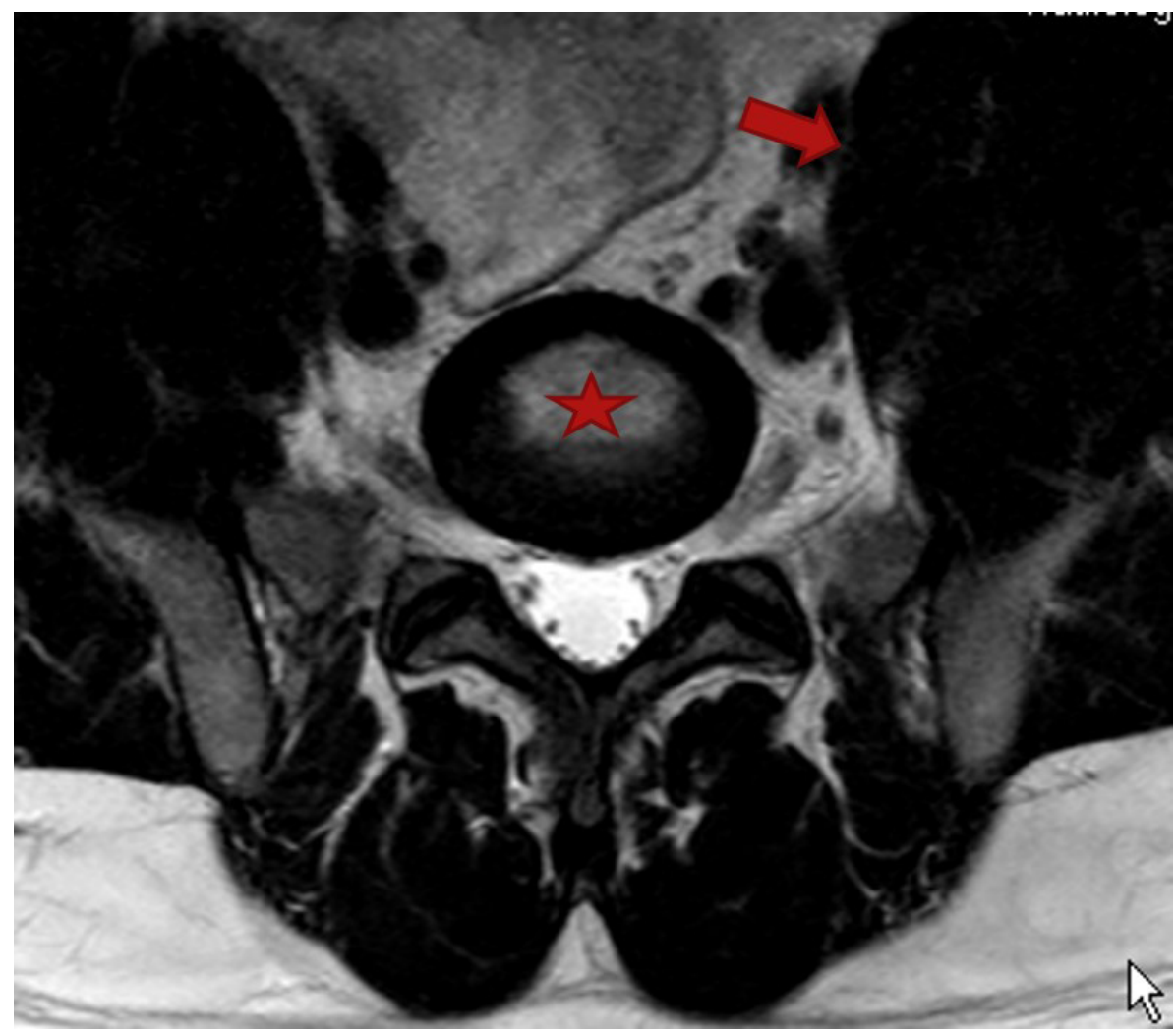

Figure 2B: MRI TSE T2W axial image of the L5-S1 disc. The PMM does not attach to the L5-S1 disc (star)

Except for few exceptions, the PMM fibers do not attach to the L5-S1 disc, as previous report affirmed a higher incidence of disc extrusion at the lower discs L4-L5 and L5-S1 [13]. Hypothetically, this may suggest a possible correlation between the absence of PMM attachment to the lower discs and the advent of disc extrusion. However, such evidences have never been confirmed nor thoroughly investigated. The focus of this study was two-fold: first, we aimed to verify the prevalence of the anatomical variants the PMM origin, and secondly to analyze the PMM attachment to the lumbar discs in correlation with disc extrusion. 


\section{Materials}

\section{Selection of participants}

MR lumbar spine examinations from the last five years - from December 2010 to December 2015 - were retrieved for the radiological archives of two Institutions: Emory University School of Medicine, Radiology Department, MSK Division Atlanta, GA, USA and Akershus University Hospital Radiology Department, MSK Division, Oslo University, Norway.

From the information obtained from the clinical charts, all patients with congenital musculoskeletal disease, history of acute trauma, acute inflammatory diseases (rheumatoid arthritis, psoriasis, gout and ankylosing spondylitis), primary neoplasia and metastases, were excluded in the first selection process. In a second round of selection, all examinations without full implementation of the MR lumbar spine examination protocol (Table 1) were excluded from the study. The same selection criteria of exclusion were used in both Institutions.

\begin{tabular}{|c|c|c|c|}
\hline Magnet & $\mathbf{1 . 5}$ Tesla & $\mathbf{1 . 5}$ Tesla & $\mathbf{1 . 5}$ Tesla \\
\hline Sequences & TSE PD & TSE T2W & TSE T2W \\
\hline Planes/ Parameters & Coronal & Axial & Sagittal \\
\hline TR & $1704 \mathrm{~ms}$ & $2333 \mathrm{~ms}$ & $3199 \mathrm{~ms}$ \\
\hline TE & $12 \mathrm{~ms}$ & $120 \mathrm{~ms}$ & $120 \mathrm{~ms}$ \\
\hline Matrix & $288 \times 224$ & $332 \times 243$ & $332 \times 243$ \\
\hline FOV & $333 \times 304$ & $200 \times 200$ & $160 \times 302$ \\
\hline Slice Thickness & $4 \mathrm{~mm}$ & $4 \mathrm{~mm}$ & $4 \mathrm{~mm}$ \\
\hline NSA & 1 & 2 & 2 \\
\hline FA & 90 degrees & 90 degrees & 90 degrees \\
\hline Number of Slices & 30 & 27 & 13 \\
\hline Examination Time & $1 \mathrm{~min} 53 \mathrm{sec}$ & 3 min $19 \mathrm{sec}$ & 3 min $21 \mathrm{sec}$ \\
\hline
\end{tabular}

Table 1: Sequences and parameters used for the MRI examination of the PMM

The selected material for the study included:1) individuals with low back pain, 2) Patients with age 15 to 85 years, both genders, 4 ) all ethnic groups. A total of 383 examinations of 213 females and 170 males met the inclusion criteria for this retrospective study. The mean age of the population was 60.3 years. The study proposal was conformed to the rules and regulations of the American Institutional Review Board (IRB) in Atlanta, Ga (USA) and the Norwegian Institutional Ethic Committee of our Institution in Oslo (Norway).

\section{Method}

\section{MR Imaging, images characteristics, and analysis of images}

All participants underwent MR examinations of the lumbar spine with inclusion of the thoracolumbar segment. The images were acquired in coronal, axial and sagittal planes. At Emory University Hospital (Atlanta GA) the images were acquired with a GE 1.5 Tesla imager. In Akershus University Hospital (Oslo), a Phillips 1.5 Tesla unit was used for the examinations. The imaging sequences and parameters from both MR units are displayed in (Table 1).

The coronal images (Figure 1) were obtained with a plan scan parallel to the long axis of the lumbar spine. The chosen field of view included the thoracolumbar junction proximally and the lumbosacral segment distally. The $4 \mathrm{~mm}$-thick coronal images displayed the vertebral bodies, the transverse processes, and the intervertebral discs. These images permitted a complete visualization of the psoas muscle from the thoracolumbar segment until the psoas reached the iliac muscle, bilaterally. The purposes of the coronal images were first, to provide an optimal assessment of the exact origin of the psoas muscle, proximally. Secondly, they offered a broad view of the muscle alongside the lumbar spine and its attachments to the vertebral bodies and to the discs on both sides. The presence or absence of attachments of the psoas was registered at each level always after verification on both coronal and axial images.

The axial images were $3 \mathrm{~mm}$-thick. These images allowed an optimal visualization of the psoas muscle and its proximity to the vertebral bodies, the transverse processes and to the discs, from Th12-L1 segment to the body of S1, distally. On these images, the muscle attachments and the anatomical variants of the attachments (absence or partial attachment) were evaluated at all levels. Similarly, the axial images were used for assessment of the intervertebral discs and disc herniation.

The sagittal images complemented the axial images. They helped to verify the exact level of the discs, vertebral bodies and the transverse processes during the image analysis. The psoas muscle was not assessed on the sagittal images. 


\section{Definition of Variants}

Variant of origin: We considered as anatomical variants of the PMM origin, when the muscle did not originate from the Th12-L1 disc level, as reported in the literature and most anatomy books. The variant of the PMM origin can be above the body of Th12 or below the body of L1.

Variant of attachment: When the psoas muscle attaches unilaterally to the vertebral body, or to the disc, or to the transverse process, it was considered as a partial attachment. However, when the psoas muscle fascicles had no visible contact with the adjacent vertebral body, transverse processes or the disc, that was registered as a total absence of attachment or anatomical variant.

\section{Statistical studies}

The prevalence of the anatomical variants of the PMM origin was calculated in the whole population (383 subjects).

In a randomly selected group of 184 subjects, the prevalence of the variants of PMM attachment to the lumbar discs was assessed, as well as the prevalence of disc extrusion.

\section{Results}

\section{Assessment of the origin of the psoas major muscle}

Among the studied population (383 examinations), the most frequent site of origin of the PMM was at the Th12-L1 disc, detected in $271(70.8 \%)$ individuals (Figures $1 \mathrm{~A}$, normal coronal image), which was considered anatomically normal in accordance with the literature.

Anatomical variants of the PMM origin were found in $112(29.2 \%)$ individuals. Of them, the PMM originated from the body of Th12 in nine (2.3\%) subjects, from the body of L1 in eighty-eight (22.9\%), from the L1-L2 disc in 12 (3.1\%), and from the body of L2 in tree $(0.7 \%)$ individuals. The psoas did not originate above the body of Th12, nor below the body of L2. We found no significant difference in the prevalence of anatomical variants of PMM origin in both genders.

The prevalence and distribution of the anatomical variants of the PMM origin are presented in (Table 2).

\begin{tabular}{|c|c|c|c|c|c|}
\hline & Site & Site & Site & Site & Total \\
\hline Genders / numbers & Th12 & L1 & L1-L2 disc & L2 & \\
\hline Females = 58 & $6(10.3)$ & $43(74.1)$ & $7(12.0)$ & $2(3.4)$ & 58 \\
\hline Males = 54 & $3(5.5)$ & $45(83.3)$ & $5(9.2)$ & $1(1.8)$ & 54 \\
\hline Total = 112 & $9(2.3)$ & $88(22.9)$ & $12(3.2)$ & $3(0.7)$ & 112 \\
\hline
\end{tabular}

Table 2: PMM origin. Prevalence and Distribution of the Anatomical Variants in 112 (29.2\%) subjects from the studied population (383 subjects)

\section{Attachment of the psoas major to the lumbar discs and prevalence of disc extrusion}

From the selected 184 examinations to assess the PMM attachment to the discs and disc extrusion, we found the PMM more frequently attached to the upper disc L1-L2 in 181 (99.4\%) subjects. The PMM was not attached to the lowest disc L5-S1 in a large percentage of the population, $173(94.02 \%)$ subjects.

There was no disc extrusion at L1-L2 level where the PMM attachment was almost constantly present (99.4\%) of individuals. The highest prevalence of disc extrusion was detected at the L5-S1 disc in 65 (35.3\%) subjects, where the PMM was less frequently attached. The correlation between the lesser frequency of PMM attachment to the lower discs L4-L5 and L5-S1 and the higher prevalence of disc extrusion at the same levels was not calculated statistically (Figure 3).

The prevalence of PMM attachment to the lumbar discs and the distribution of disc extrusion in the selected population of 184 subjects are displayed in Table 3.

\begin{tabular}{|c|c|c|c|c|c|}
\hline Total $=\mathbf{1 8 4}$ & Site & Site & Site & Site & Total \\
\hline $\begin{array}{c}\text { PMM } \\
\text { Attachment } \\
\text { to the disc }\end{array}$ & $181(99.4 \%)$ & $179(97.2 \%)$ & $182(98.9 \%)$ & $144(78.3)$ & $11(6.0 \%)$ \\
\hline $\begin{array}{c}\text { Disc Extrusion } \\
\text { Detected }\end{array}$ & $0(100 \%)$ & $4(2.2 \%)$ & $29(15.8 \%)$ & $62(33.7 \%)$ & $65(35 \%)$ \\
\hline
\end{tabular}

Table 3: Prevalence and Distribution of PMM attachment at the discs and presence of disc extrusion at the same levels in the selected 184 subjects 


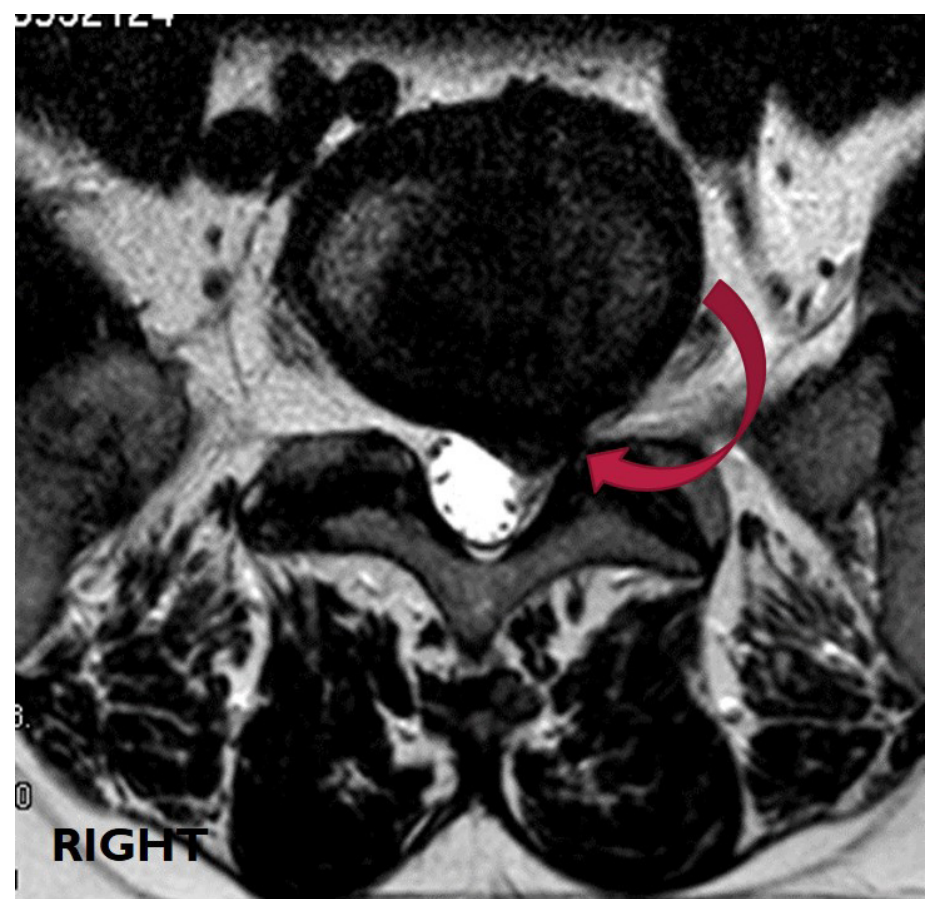

Figure 3A: MRI TSE T2W axial image of the L5-S1 disc of a patient with back pain and paresthesia in the left lower extremity. The image shows a large disc extrusion on the left (curved arrow). There is compression of the left nerve root S1. Note the absence of PMM attachment to the disc

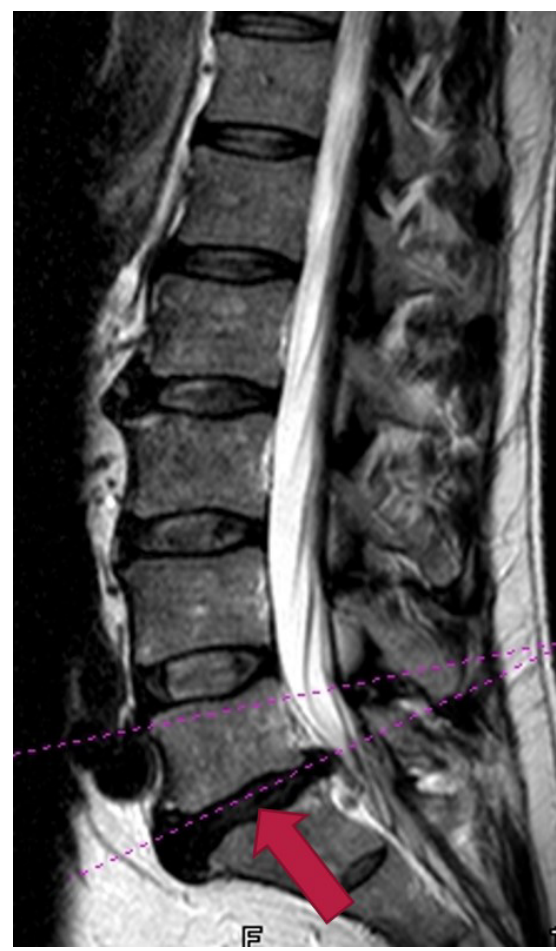

Figure 3B: (Same patient as in figure 3A). MRI TSE T2W sagittal image of the lumbar spine. The dotted line (arrow) indicates the plan scan through the extruded disc L5-S1

\section{Discussion}

The retrospective analysis of magnetic resonance (MR) images of the lumbar spine revealed a relatively high prevalence of anatomical variants of the PMM origin (29.2\%). The anatomical origin of the PMM at the Th12-L1 disc was already reported [1$4,14]$, however the prevalence of the anatomical variants of the muscle origin has not been demonstrated. Hypothetically, because of the role of the PMM as a stabilizer it is not certain whether these anatomical variations can alter the biomechanics of the lumbar spine. In the selection of the studied population, all individuals with congenital diseases of the musculoskeletal system were excluded. Despite these precautions, a high prevalence of anatomical variants of the PMM origin was detected. That suggests the non-pathological nature of these variants, and that they are by no means related to any known systemic disorders of the skeletal muscles. 
The second focus of this study related to the PMM attachment to the lumbar discs. Previous anatomy reports stated the PMM closely attached to both sides of the discs along the lumbar spine [1-4,14,15]. In this study, we found the PMM attached to the upper discs from L1 to L4 in most of the selected group of 184 individuals. However, there was an almost constant absence of attachment to the lower disc L5-S1. Likewise, disc extrusion was present at a higher prevalence at L5-S1 compared with the upper discs. This coincidence has not been reported earlier.

It is not sure whether such finding could suggest that the PMM attachment to the outer sides of the upper discs would strengthen the annulus fibrosus and prevent the nucleus pulposus from extruding. Neither could we find any study affirming that the lack of the PMM attachment to the lower discs would contribute to a higher incidence of disc extrusion. Previous studies of the skeletal muscle [15-19] focused on the increased pressure in the intervertebral disc at the lumbar spine in sitting position as prejudicial to the disc. However, the partial or total absent of PMM attachment to the lower discs has never been considered or proposed as a predisposing factor in disc extrusion.

This study carried some weakness. First, it was a retrospective analysis. Secondly, the distribution and prevalence of the anatomical variants in various ethnic groups were not calculated. Thirdly, the correlation between the absence of PMM attachment to the lowers discs and the high prevalence of disc extrusion at the same levels was not statistically assessed. Also, in this study the laterality of the disc herniation and its relation with the site of attachment of the muscle has not been investigated, which deserves further attention.

\section{Conclusion}

In conclusion, this study confirmed the PMM to originate most frequently from the Th12-L1 disc. Anatomical variants of the PMM origin were found from Th12 to L2, but never above Th12 or below L2. In most individuals, the PMM did not attach to the L5-S1 disc; and disc extrusion occurred more frequent at L5-S1 level. Further investigations are needed to determine whether this was a merely coincidence or a cause-effect phenomenon.

\section{References}

1. Gray H, Standring S (2008) Gray's Anatomy -The Anatomical Basis of Clinical Practice, Expert Consult ( $40^{\text {th }}$ Edn) Churchill Livingstone-Elsevier.

2. Kopsch F. (1908) Rauber's Lehrbuch der Anatomie des Menschen (10 $10^{\text {th }}$ Edn) Georg Thieme, Stuttgart Leipzig.

3. Bogduk N, Pearcy M, Hadfield G (1992) Anatomy and biomechanics of psoas major. Clin Biomech 7: 109-19.

4. Arbanas J, Klasan GS, Nikolic M, Jerkovic R, Miljanovic I, et al. (2009) Fibre type composition of the human psoas major muscle with regard to the level of its origin. J Anat 215: 636-41.

5. Andersson E, Oddsson L, Grundström H, Thorstensson A (1995) The role of the psoas and iliacus muscles for stability and movement of the lumbar spine, pelvis and hip. Scand J Med Sci Sports 5: 10-6.

6. Arbanas J, Pavlovic I, Marijancic V, Vlahovic H, Starcevic-Klasan G, et al. (2013) MRI features of the psoas major muscle in patients with low back pain. Eur Spine J 22: 1965-71.

7. Skyrme AD, Cahill DJ, Marsh HP, Ellis H (1999) Psoas Major and its Controversial Rotational Action. Clin Anat 12: 264-5.

8. Penning L (2000) Psoas muscle and lumbar spine stability: a concept uniting existing controversies. Critical review and hypothesis. Eur Spine J 9: 577-85.

9. Gracovetsky S (1985) An hypothesis for the role of the spine in human locomotion: a challenge to current thinking. J Biomed Eng 7: 205-16.

10. Hansen L, de Zee M, Rasmussen J, Andersen TB, Wong C, et al. (2006) Anatomy and biomechanics of the back muscles in the lumbar spine with reference to biomechanical modeling. Spine 31: 1888-99.

11. Pearcy M, Portek I, Shepherd J (1984) Three-dimensional x-ray analysis of normal movement in the lumbar spine. Spine 9: 294-7.

12. Sajko S, Stuber K (2009) Psoas Major: a case report and review of its anatomy, biomechanics and clinical implications. J Can Chiropr Ass 53: 311-8.

13. Keagy RD, Brumlik J, Bergan JL (1966) Direct electromyography of the psoas major muscle in man. J Bone Joint Surg Am 48: 1377-82.

14. Yoshio M, Murakami G, Sato T, Sato S, Noriyasu S (2002) The function of the psoas major muscle: passive kinetics and morphological studies using donated cadavers. J Orthop Sci 7: 199-207.

15. Nachemson AL (1981) Disc pressure measurements. Spine 6: 93-7.

16. Schultz A, Andersson G, Ortengren R, Haderspeck K, Nachemson A (1982) Loads on the lumbar spine. Validation of a biomechanical analysis by measurements of the intradiscal pressure andmyoelectric signals. J Bone Joint Surg Am 64: 713-20.

17. Smidt GL (1993) Sitting pressure in a waiting room environment. Clin Biomech 9: 323-4.

18. Wilke HJ, Neef P, Caimi M, Hoogland T, Claes LE (1999) New in vivo measurements of pressures in the intervertebral disc in daily life. Spine 24: 755-62.

19. Liu Y, Kong X, Li F, Tan B, Li Y, et al. (2016) Co-dependence of genotype and dietary protein intake to affect expression on amino acid/peptide transporters in porcine skeletal muscle. Amino Acids 48: 75-90. 


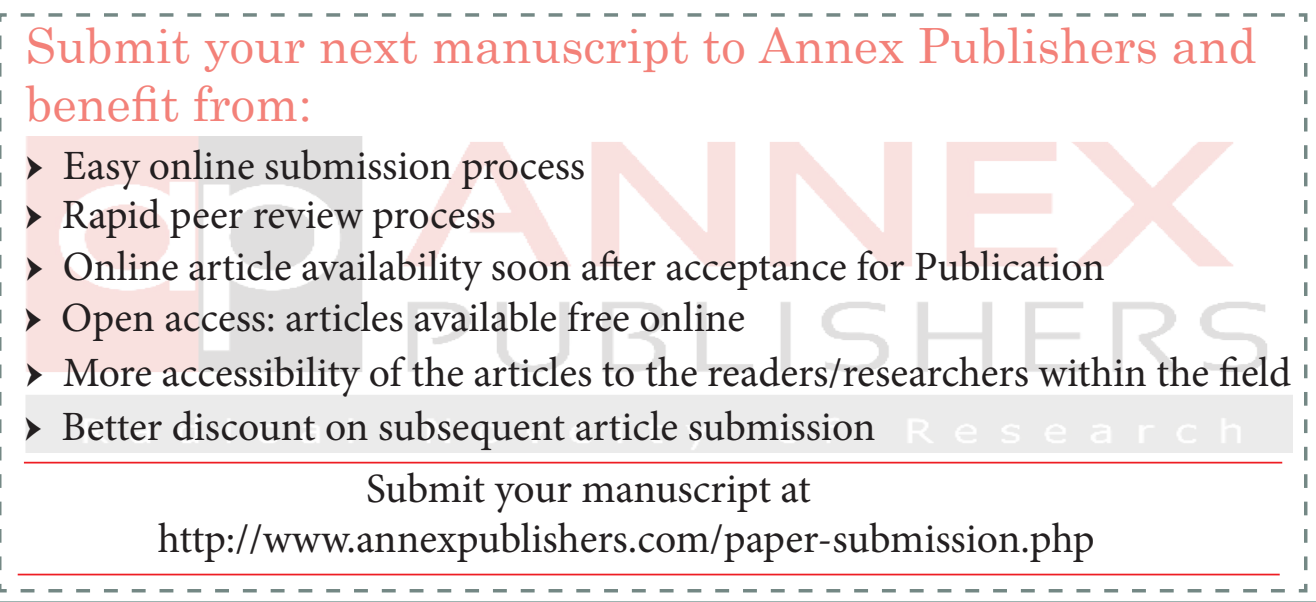

